PROCEEDINGS OF THE

AMERICAN MATHEMATICAL SOCIETY

Volume 139, Number 10, October 2011, Pages 3445-3450

S 0002-9939(2011)10809-8

Article electronically published on March 4, 2011

\title{
ALMOST REGULAR INVOLUTORY AUTOMORPHISMS OF UNIQUELY 2-DIVISIBLE GROUPS
}

\author{
YOAV SEGEV \\ (Communicated by Jonathan I. Hall)
}

\begin{abstract}
We prove that a uniquely 2-divisible group that admits an almost regular involutory automorphism is solvable.
\end{abstract}

\section{INTRODUCTION}

Recall that an automorphism $\nu$ of a group $H$ is called involutory if $\nu \neq i d$ and $\nu^{2}=i d$. The automorphism $\nu$ is called almost regular if $C_{H}(\nu)$ is finite. Recall that a group $U$ is uniquely 2-divisible if for each $u \in U$ there exists a unique $v \in U$ such that $v^{2}=u$. Note that in particular a uniquely 2-divisible group contains no involutions (i.e. elements of order 2).

The purpose of this paper is to use the techniques introduced in the impressive paper $[\mathrm{Sh}$ of Shunkov, where he proves that a periodic group that admits an almost regular involutory automorphism is virtually solvable (i.e. it has a solvable subgroup of finite index). We prove

Theorem 1.1. Let $U$ be a uniquely 2-divisible group. If $U$ admits an involutory almost regular automorphism, then $U$ is solvable.

Our main motivation for dealing with automorphisms of uniquely 2-divisible groups comes from questions about the root groups of special Moufang sets, and those tend to be uniquely 2-divisible; see, e.g., [S]. Indeed, using Theorem 1.1 it immediately follows that

Corollary 1.2. Let $\mathbb{M}(U, \tau)$ be a special Moufang set. If the Hua subgroup contains an involution $\nu$ such that $C_{U}(\nu)$ is finite, then $U$ is abelian.

Proof. If $U$ contains involutions, then $U$ is abelian by [DST, Theorem 5.5, p. 782]. If $U$ does not contain involutions, then by [DS, Proposition 4.6, p. 5840], $U$ is uniquely 2-divisible, and then by Theorem 1.1 and by the main theorem of [SW], $U$ is abelian.

The proof of Theorem 1.1 is obtained as follows. First note that if $U$ is finite, then $U$ has odd order, so by the Feit-Thompson theorem $U$ is solvable. Hence we may assume that $U$ is infinite.

Received by the editors September 1, 2010.

2010 Mathematics Subject Classification. Primary 20E36.

Key words and phrases. Almost regular involutory automorphism, uniquely 2-divisible group.

(C)2011 American Mathematical Society Reverts to public domain 28 years from publication 3445 
We let $A$ be a maximal abelian subgroup of $U$ (with respect to inclusion) inverted by $\nu$ (i.e. each element of $A$ is inverted by $\nu$ ). In Lemma 3.1(2) we show that we can take $A$ to be infinite. We then show that for elements $u_{1}, \ldots, u_{n} \in U$, the involutions $u_{1} \nu u_{1}^{-1}, \ldots, u_{n} \nu u_{n}^{-1}$ in the semi-direct product $U \rtimes\langle\nu\rangle$ invert a subgroup $D \leq A$ with $|A: D|<\infty$ (Proposition 3.3). The next step is to show that $C_{U}(D) / D$ is finite and solvable (Lemma 3.5). Since $K:=\left\langle\nu u_{1} \nu u_{1}^{-1}, \ldots, \nu u_{n} \nu u_{n}^{-1}\right\rangle \leq C_{U}(D)$, the subgroup $K$ is solvable and $K / Z(K)$ is finite.

Next let $S:=\left\{x \in U \mid x^{\nu}=x^{-1}\right\}$. It is easy to see that an element $y \in U$ is in $S$ iff $y=\nu u \nu u^{-1}$, for some $u \in U$, so by the above each finitely generated subgroup $H$ of $R:=\langle S\rangle$ is solvable and satisfies the fact that $H / Z(H)$ is finite. It follows that $R^{\prime}$ is periodic (Proposition 3.6). Using the above mentioned result of Shunkov, we see that $R^{\prime}$ is solvable, so $R$ is solvable.

As is well known (see [ $\mathrm{K}]$ ), $U=R C_{U}(\nu)$ and $R \unlhd U$. Since $C_{U}(\nu)$ is finite and uniquely 2-divisible it has odd order. By the Feit-Thomson theorem, $C_{U}(\nu)$ is solvable, and this at last shows that $U$ is solvable.

We remark that it is possible that with the aid of the Theorem on page 286 of $[\mathrm{HM}$, one can get even more delicate information on $U$. However, we do not need that, so we do not pursue this avenue further.

\section{NOTATION AND PRELIMINARY RESUltS}

Notation 2.1. (1) Throughout this paper $U$ is an infinite uniquely 2-divisible group and $\nu \in \operatorname{Aut}(U)$ is an involutory automorphism which is almost regular.

(2) We denote by $G$ the semi-direct product of $U$ by $\nu$, and we identify $U$ and $\nu$ with their images in $G$. We let $\operatorname{Inv}(G)$ denote the set of involutions of $G$.

(3) We let $S:=\left\{x \in U \mid x^{\nu}=x^{-1}\right\}$.

(4) The letter $A$ always denotes a fixed infinite maximal (with respect to inclusion) abelian subgroup of $U$ which is inverted by $\nu$ (i.e. all of whose elements are inverted by $\nu$ ). The existence of $A$ is guaranteed by Lemma 3.1(2) and by Zorn's lemma.

(5) For each $u \in U$ we denote by $A_{u}$ the subgroup of $A$ inverted by $u \nu u^{-1}$.

Remark 2.2. (1) Note that for any non-empty subset $T \subseteq U$, the centralizer $C_{U}(T)$ is a uniquely 2-divisible subgroup of $U$.

(2) Notice that $A$ is uniquely 2-divisible. Also, for any $u \in U$, the subgroup $A_{u}$ is uniquely 2 -divisible.

(3) It is easy to check that $S=\left\{\nu \nu^{x} \mid x \in U\right\}$.

Lemma 2.3 ( $[\mathrm{N}]$, Lemma 4.1, p. 239). Let the group $H$ be the union of finitely many, let us say $n$, cosets of subgroups $C_{1}, C_{2}, \ldots, C_{n}$ :

$$
H=\bigcup_{i=1}^{n} C_{i} g_{i} \text {. }
$$

Then the index of (at least) one of these subgroups in $H$ does not exceed $n$.

Corollary 2.4. Let the group $H$ be the union of finitely many, let us say $n$, subsets $S_{1}, S_{2}, \ldots, S_{n}$ :

$$
H=\bigcup_{i=1}^{n} S_{i}
$$

For each $i$ set $C_{i}:=\left\langle a b^{-1} \mid a, b \in S_{i}\right\rangle$. Then the index of (at least) one of the subgroups $C_{1}, \ldots, C_{n}$ in $H$ does not exceed $n$. 
Proof. For each $i=1, \ldots, n$, pick an arbitrary $g_{i} \in S_{i}$. Notice that $S_{i} \subseteq C_{i} g_{i}$ for all $i$, so $H=\bigcup_{i=1}^{n} C_{i} g_{i}$ and Corollary 2.4 follows from Lemma 2.3

Lemma 2.5. (1) All involutions in $G$ are conjugate;

(2) $S=\{\nu \tau \mid \tau \in \operatorname{Inv}(G)\}$.

Proof. Let $\tau \in \operatorname{Inv}(G)$. Then $\tau=x \nu$ for some $x \in U$. Since $\tau$ is an involution, $x \in S$. Let $y \in U$ be the unique element with $y^{2}=x$. Then $y \in S$ and $\tau=x \nu=$ $y^{2} \nu=y \nu y^{-1}$. This shows (1). Part (2) is Remark 2.2(3).

Lemma 2.6. Let $D$ be an abelian uniquely 2-divisible subgroup of $U$. Then the following hold:

(1) $C_{U}(D) / D$ is a uniquely 2-divisible group.

(2) If $D$ is inverted by $\nu$, then $\nu D$ is an almost regular involutory automorphism of $C_{U}(D) / D$.

(3) Assume that $D$ is inverted by $\nu$ and let $E / D$ be a subgroup of $C_{U}(D) / D$ which is inverted by $\nu D$. Then $E$ is inverted by $\nu$, so, in particular, $E$ is abelian.

Proof. (1) Set $C:=C_{U}(D)$. Assume that $a, b \in C$ and $a^{2} D=b^{2} D$. Let $x, y \in D$ with $a^{2} x=b^{2} y$ and let $u, v \in D$ with $u^{2}=x$ and $v^{2}=y$. Then $a^{2} u^{2}=b^{2} v^{2}$, and since $a, b$ commute with $u, v$ we see that $(a u)^{2}=(b v)^{2}$. Hence $a u=b v$, so $a D=b D$.

Furthermore let $a D \in C / D$. Let $b \in U$ with $b^{2}=a$. Then $b \in C$ and $b D$ is the square root of $a D$ in $C / D$.

(2) Clearly $\nu D$ is an involutory automorphism of $C / D$ (acting via conjugation). Assume that $a D \in C / D$ centralizes $\nu D$. Then $\nu^{a}=\nu d$ for some $d \in D$. Let $x \in D$ with $x^{2}=d$. Then $\nu$ inverts $x$, and we see that $\nu^{a}=\nu^{x}$ and $a x^{-1} \in C_{U}(\nu)$. It follows that $C_{C / D}(\nu D)=C_{C}(\nu) D / D$, and since $\nu$ is almost regular, so is $\nu D$.

(3) Let $x D \in C / D$ be an element inverted by $\nu D$. Then $x^{\nu}=x^{-1} d$ for some $d \in D$, and conjugating by $\nu$ we see that $x=x^{-\nu} d^{-1}$, which implies that $x^{\nu}=x^{-1} d^{-1}$. Thus $d=d^{-1}$, so $d=1$.

Now let $e \in E$. Then, by hypothesis, $e D$ is inverted by $\nu D$, so $e^{\nu}=e^{-1}$.

\section{The Proof of Theorem 1.1}

Lemma 3.1. Let $D$ be an abelian subgroup of $U$ (we allow $D=1$ ) such that $D$ is inverted by $\nu$ and such that $C_{U}(D)$ is infinite. Assume that

$$
\left(S \cap C_{U}(D)\right) \backslash D \neq \emptyset .
$$

Then the following hold:

(1) There exists an element $w \in C_{U}(D) \backslash D$ which is inverted by $\nu$ and such that $C_{U}(\langle D, w\rangle)$ is infinite.

(2) There exists an infinite abelian subgroup of $U$ which is inverted by $\nu$.

Proof. (1) Set $V:=C_{U}(D)$. Then $V$ is an infinite uniquely 2-divisible group, and $\nu$ acts on $V$, so without loss we may assume that $U=V$ and that $D \leq Z(U)$.

Pick $b \in S \backslash D$ (note that $b$ exists by hypothesis), and write $b=\nu \tau$ with $\tau \in \operatorname{Inv}(G)$. Let

$$
u \in U \text { with } u^{-2}=\nu \tau,
$$


and note that since $u$ is inverted by both $\nu$ and $\tau$, we have

$$
\nu=\tau^{u} \text {. }
$$

We claim that there exists $h \in C_{U}(\tau)$ such that $h u$ is inverted by infinitely many involutions of $G$. Suppose for a moment that the claim holds. Note that $h u \notin D$, for all $h \in C_{U}(\tau)$. Indeed, if $h=1$, then $h u=u$, and since $b \notin D$ also $u \notin D$. Otherwise if $h u \in D$ and $h \neq 1$, then

$$
u^{-1} h^{-1}=(h u)^{\tau}=h^{\tau} u^{\tau}=h u^{-1},
$$

and it follows that $u$ inverts $h$, which is not possible in a uniquely 2-divisible group.

Since all involutions in $G$ are conjugate, conjugating $h u$ by an appropriate element we may assume that $\nu$ inverts $h u$, and since $h u$ is inverted by infinitely many involutions, we see that $C_{U}(h u)$ is infinite. Taking $w=h u$, we are done.

It remains to show the existence of $h$. For each $a \in S$, let

$$
s_{a}:=\nu \tau^{a} \text { and } \ell_{a}^{-2}=s_{a} .
$$

It is easy to check that since $\ell_{a}$ is inverted by $\nu$ and $\tau^{a}$, we have $\tau^{a \ell_{a}}=\nu$. Hence

$$
\tau^{a \ell_{a}}=\tau^{u} \text {, and hence } h_{a}:=a \ell_{a} u^{-1} \in C_{U}(\tau) .
$$

It follows that $\ell_{a}=a^{-1} h_{a} u$. Since both $\ell_{a}$ and $a$ are inverted by $\nu$ we get after conjugating by $\nu$ that $\ell_{a}^{-1}=a\left(h_{a} u\right)^{\nu}=\left(h_{a} u\right)^{-1} a$. Notice now that $a \nu \in \operatorname{Inv}(G)$, and it follows that

$$
\left(h_{a} u\right)^{a \nu}=\left(h_{a} u\right)^{-1} .
$$

By hypothesis the set $\left\{h_{a} \mid a \in S\right\}$ is finite since it is contained in $C_{U}(\tau)$. Further, the set $S$ is infinite. This implies the existence of $h \in C_{U}(\tau)$ such that the number of involutions $a \nu$ that invert $h u$ is infinite. This proves (1).

(2) If $D$ is finite and $C_{U}(D)$ is infinite, then, since $\nu$ is almost regular, $(S \cap$ $\left.C_{U}(D)\right) \backslash D \neq \emptyset$ (because $S \cap C_{U}(D)$ is infinite; see Remark 2.2(3)). Hence (2) follows from (1) by starting with $D=1$ and iterating the process as long as the subgroup $\langle D, w\rangle$ is finite.

Lemma 3.2. Let $x \in U$ and let $s \in U$ be the unique element such that $s^{-2}=$ $\nu x^{-1} \nu x$. Then $x s \in C_{U}(\nu)$.

Proof. Notice that $s$ is inverted by $\nu$ and $\nu^{x}$. Hence

$$
1=s^{2} \nu \nu^{x}=\nu s^{-2} \nu^{x}=\nu s^{-1} \nu^{x} s,
$$

so the lemma holds.

Proposition 3.3. Let $A$ be as in Notation 2.1(4) and let $u \in U$. Let $A_{u}$ be as in Notation 2.1(5). Then $\left|A: A_{u}\right|<\infty$.

Proof. Fix $a \in A$ and consider the element

$$
\nu \nu^{a u} \text {. }
$$

This element is in $U$. Let $s \in U$ with $s^{-2}=\nu \nu^{a u}$. By Lemma 3.2 we get that

$$
v_{a}:=\text { aus } \in C_{U}(\nu) \text {. }
$$

Now set

$$
\mathcal{M}_{a}:=\left\{b \in A \mid v_{b}=v_{a}\right\} .
$$

Notice that since $\left|C_{U}(\nu)\right|<\infty$,

$$
\text { the set }\left\{\mathcal{M}_{c} \mid c \in A\right\} \text { is finite and } A=\bigcup_{c \in A} \mathcal{M}_{c} \text {. }
$$


By equation (3.1) we get $s^{-1}=v_{a}^{-1} a u$, and conjugating by $\nu$ and noticing that $\nu$ inverts $a$ and $s$ and centralizes $v_{a}$, we see that $s^{-1}=u^{-\nu} a v_{a}$. So we get the equality

$$
v_{a}^{-1} a u=u^{-\nu} a v_{a},
$$

from which it follows that

$$
u^{-1} \nu b v_{a} u^{-1}=\nu v_{a}^{-1} b, \quad \forall b \in \mathcal{M}_{a} .
$$

Let $c \in \mathcal{M}_{a}$. Then as in equation (3.3) we get that $u^{-1} \nu c v_{a} u^{-1}=\nu v_{a}^{-1} c$, and this together with equation (3.3) yields

$$
u v_{a}^{-1} c^{-1} b v_{a} u^{-1}=c^{-1} b, \quad \forall b, c \in \mathcal{M}_{a} .
$$

Since $\nu$ inverts $c^{-1} b \in A$, it follows that $u v_{a}^{-1} \nu v_{a} u^{-1}=u \nu u^{-1}$ inverts $c^{-1} b$. We thus can conclude that

$$
u \nu u^{-1} \text { inverts }\left\langle b c^{-1} \mid b, c \in \mathcal{M}_{a}\right\rangle, \quad \forall a \in A .
$$

By equation (3.2) and by Corollary 2.4 one of the subgroups $\left\langle b c^{-1} \mid b, c \in \mathcal{M}_{a}\right\rangle$ has finite index in $A$, so $\left|A: A_{u}\right|<\infty$ as asserted.

Lemma 3.4. Let $B$ be a finitely generated abelian subgroup of $U$ which is inverted by $\nu$. Then $A$ contains a subgroup $A_{1}$ of finite index such that $\left\langle A_{1}, B\right\rangle$ is abelian.

Proof. Recall the definition of $A$ from Notation 2.1(4) and for $b \in B$ the definition of $A_{b}$ from Notation 2.1(5). Let $\mathcal{B}$ be a finite set of generators for $B$ and set $A_{1}:=\bigcap_{b \in \mathcal{B}} A_{b}$. By Proposition 3.3 and since $\mathcal{B}$ is finite, $\left|A: A_{1}\right|<\infty$. Further, for each $b \in \mathcal{B}, \nu$ and $b \nu b^{-1}$ invert $A_{1}$, so $b^{2}=b \nu b^{-1} \nu \in C_{U}\left(A_{1}\right)$ (recall that $\nu$ inverts b). Since $U$ is uniquely 2-divisible, $b \in C_{U}\left(A_{1}\right)$. Hence $\mathcal{B} \leq C_{U}\left(A_{1}\right)$ and the lemma holds.

Lemma 3.5. Let $D$ be a uniquely 2-divisible subgroup of $A$ of finite index. Then $C_{U}(D) / D$ is finite and solvable.

Proof. Set $C:=C_{U}(D)$ and $\bar{C}:=C / D$. Assume that $\bar{C}$ is infinite. By Lemma2.6(1), $\bar{C}$ is uniquely 2-divisible, and by hypothesis $\bar{A}:=A / D$ is a finite subgroup of $\bar{C}$.

Let $\overline{\mathcal{A}}$ be an infinite maximal abelian subgroup of $\bar{C}$ inverted by $\nu D$. The existence of $\overline{\mathcal{A}}$ is guaranteed by Lemma 2.6(2) and by Lemma 3.1(2) (with $\bar{C}$ in place of $U$ ). By Lemma 3.4 (with $\bar{C}$ in place of $U$ and $\bar{A}$ in place of $B$ ), there exists a finite index $\overline{\mathcal{A}_{1}} \leq \overline{\mathcal{A}}$ such that $\overline{\mathcal{A}_{2}}:=\left\langle\overline{\mathcal{A}_{1}}, \bar{A}\right\rangle$ is abelian. Note that $\overline{\mathcal{A}_{2}}$ is inverted by $\nu D$, so by Lemma $2.6(3)$ the inverse image $\mathcal{A}_{2}$ of $\overline{\mathcal{A}_{2}}$ in $C_{U}(D)$ is an abelian subgroup inverted by $\nu$. Clearly $\mathcal{A}_{2}$ properly contains $A$. This contradicts the maximality of $A$ and shows that $\bar{C}$ is finite.

Suppose $t D \in \bar{C}$ is an involution. Then $t^{2} \in D$, so also $t \in D$, and we see that $\bar{C}$ has odd order. By the Feit-Thompson theorem, $\bar{C}$ is solvable, and the proof of the lemma is complete.

Proposition 3.6. Let $R:=\langle S\rangle$. Then:

(1) $R^{\prime}$ is a periodic group;

(2) $R$ is solvable. 
Proof. (1) We first show that for elements $u_{1}, \ldots, u_{n} \in U$ the subgroup $K:=$ $\left\langle\nu u_{1} \nu u_{1}^{-1}, \ldots, \nu u_{n} \nu u_{n}^{-1}\right\rangle$ is solvable and $K / Z(K)$ is finite. By Remark $2.2(3)$, this will show that

(*) if $H$ is a finitely generated subgroup of $R$,

then $H$ is solvable, and $H / Z(H)$ is finite.

Let $D:=\bigcap_{i=1}^{n} A_{u_{i}}$. By the definition of $A_{u_{i}}$ and by Proposition 3.3, $|A: D|<\infty$ and $D$ is inverted by $\nu, u_{1} \nu u_{1}^{-1}, \ldots, u_{n} \nu u_{n}^{-1}$. Also, by Remark 2.2 (2), $D$ is uniquely 2-divisible. By Lemma 3.5 $C_{U}(D) / D$ is finite and solvable, so since $K \leq C_{U}(D)$, we see that $K / Z(K)$ is finite and solvable. Hence $(*)$ holds.

Next let $g \in R^{\prime}$. Then there exists a finitely generated subgroup $H$ of $R$ such that $g \in H^{\prime}$. By $(*)$ and by [A, (33.9), p. 168], $H^{\prime}$ is finite, so the order of $g$ is finite. This completes the proof of part (1).

(2) By (1), $R^{\prime}$ is a periodic group, and since $R$ is $\nu$-invariant, $\nu$ is an almost regular automorphism of $R^{\prime}$. By the main result of Shunkov in $\underline{\mathrm{Sh}}, R^{\prime}$ is virtually solvable. But by $(*), R^{\prime}$ is also locally solvable, so this shows that $R^{\prime}$ is solvable and hence so is $R$.

Proof of Theorem 1.1. By Proposition [3.6, $\langle S\rangle$ is solvable. By [K, (3.4), p. 281] (see also [S, Lemma 2.1(1) and Lemma 2.2(1)]), $U=\langle S\rangle C_{U}(\nu)$ and $\langle S\rangle \unlhd U$. Since $C_{U}(\nu)$ is a finite uniquely 2-divisible group, it has odd order. By the Feit-Thompson theorem it is solvable. Hence $U$ is solvable.

\section{ACKNOWLEDGMENTS}

The author would like to thank Pavel Shumyatsky for several fruitful email exchanges. Thanks are also due to Richard Weiss for several comments that improved the exposition in this paper.

\section{REFERENCES}

[A] M. Aschbacher, Finite group theory, Cambridge Studies in Advanced Mathematics, 10. Cambridge University Press, Cambridge, 1986. MR.895134 (89b:20001)

[DS] T. De Medts, Y. Segev, Identities in Moufang sets, Trans. Amer. Math. Soc. 360 (2008), no. 11, 5831-5852. MR2425693 (2009f:20042)

[DST] T. De Medts, Y. Segev, K. Tent, Special Moufang sets, their root groups and their $\mu$-maps, Proc. Lond. Math. Soc. (3) 96 (2008), no. 3, 767-791. MR2407819 (2009d:20068)

[HM] B. Hartley, Th. Meixner, Periodic groups in which the centralizer of an involution has bounded order, J. Algebra 64 (1980), no. 1, 285-291. MR575796 (81i:20049)

[K] L. G. Kovács, Groups with regular automorphisms of order 4, Math. Zeitschr. 75 (1961), 277-294. MR0123613 (23:A938)

[N] B. H. Neumann, Groups covered by permutable subsets, J. London Math. Soc. 29 (1954), 236-248. MR0062122 (15:931b)

[S] Y. Segev, Toward the abelian root groups conjecture for special Moufang sets, Advances in Mathematics 223 (2010), 1545-1554. MR2592502

[SW] Y. Segev, R. Weiss, On the action of the Hua subgroups in special Moufang sets, Math. Proc. Cambridge Philos. Soc. 144 (2008), no. 1, 77-84. MR2388234 (2009a:20002)

[Sh] V. P. Shunkov, Periodic groups with an almost regular involution, Algebra and Logic 11 (1974), 260-272.

Department of Mathematics, Ben-Gurion University, Beer-Sheva 84105, Israel

E-mail address: yoavs@math.bgu.ac.il 